\title{
Arquitetura situacional do crédito: Tempo, cognição, afeto e decisão
}

The Situational Architecture of Credit: Time, Cognition, Affect and Decisionmaking

Architecture situationnelle du crédit: temps, cognition, affection et décision

Miguel Oliveira e Fernanda Jesus

\section{CpenEdition}

\section{Journals}

Edição electrónica

URL: http://journals.openedition.org/rccs/5356

DOI: $10.4000 /$ rccs. 5356

ISSN: 2182-7435

Editora

Centro de Estudos Sociais da Universidade de Coimbra

Edição impressa

Data de publição: 1 setembro 2013

Paginação: 39-64

ISSN: 0254-1106

Refêrencia eletrónica

Miguel Oliveira e Fernanda Jesus, «Arquitetura situacional do crédito: Tempo, cognição, afeto e decisão », Revista Crítica de Ciências Sociais [Online], 101 | 2013, posto online no dia 17 fevereiro 2014 consultado o 04 fevereiro 2021. URL: http://journals.openedition.org/rccs/5356 ; DOI: https://doi.org/ $10.4000 /$ rccs. 5356 


\section{MIGUEL OLIVEIRA FERNANDA JESUS}

\section{Arquitetura situacional do crédito: Tempo, cognição, afeto e decisão*}

Propomo-nos respigar neste artigo os elementos situacionais que na arquitetura do crédito assumem comprovada ou hipoteticamente o papel de fatores determinantes das preferências e escolhas, bem como descrever e ilustrar os elementos psicológicos, muitos dos quais já testados em diversos paradigmas de pesquisa, que, em operação no decisor, com aqueles interagem. Procuramos mostrar que, na circunstância característica do crédito, embora correspondendo à situação intertemporal clássica e aos comportamentos padrão que ela suscita (e.g. enviesamento do presente), intervêm outras peculiaridades que resultam do funcionamento do complexo cognitivo-afetivo do decisor, pautado por uma indeterminação e instabilidade funcionais. Anotamos como estas revertem, finalmente, num comportamento adaptativo cujas consequências podem afastar-se do melhor interesse do decisor.

Palavras-chave: comportamento do consumidor; crédito ao consumidor; finanças pessoais; instabilidade funcional; tomada de decisão.

Often is best to ask people to take care of themselves, but when people borrow, standard human frailties can lead to serious hardship and even disaster.

Thaler e Sunstein (2008: 144)

It's disturbing to think of yourself as so fluid, so potentially unstable, held together only by the shifting influence of available rewards. It's like being told that atoms are mostly empty and wondering how they can bear weight. Yet the bargaining of interests in a society can produce highly stable institutions; perhaps that's also true of the internal interests created by a person's rewards.

AinsLIE (2001: 44)

* Parte do trabalho apresentado neste artigo foi realizado no âmbito do Projeto BEHAVE (PTDC/ PSI-PSO/114257/2009 - FCOMP-01-0124-FEDER-015552), financiado pela Fundação para a Ciência e a Tecnologia. 


\section{Introdução}

No atual momento sociopolítico e económico, uma abordagem sociopsicológica sobre os processos envolvidos na construção das preferências e atitudes face ao crédito poderá ressumar como demasiado tardia ou, num tom mais compassivo, como cavalgando o benefício da retrospetiva. Fazer, como propomos, uma viagem atenta pela literatura e pesquisa sobre a construção de atitudes face ao consumo e ao crédito poderá deixar uma impressão de mescla de ordem e emaranhamento. Esta impressão cremos que se dissipará à medida que damos o passo em direção à reflexão simples que consiste no cotejo das coisas destiladas da literatura especializada com as incidências que a arquitetura do crédito impõe em exemplos da vida do dia a dia.

\section{Crédito e consumo}

Se o crédito, tal como hoje nos surge, parece ser um fenómeno do momento presente, ele não deixava já de figurar numa previsão bem incisiva sobre a sua função numa sociedade de consumo como é a nossa. Baudrillard vaticinava há algumas décadas que a sua eminência era óbvia:

O consumidor 'a crédito' aprende pouco a pouco a usar com toda a liberdade o objeto como se fosse 'seu'. O 'vencimento' do objeto está ligado à sua degradação... Isto implica sempre o risco, em caso de incumprimento ou perda, de que o objeto esteja deteriorado antes de se vencer o pagamento. Este risco define, aí mesmo onde ele parece perfeitamente integrado na vida quotidiana, uma insegurança que não foi nunca a do objeto 'patrimonial'. Este último é meu: está pago. O objeto a crédito será meu quando 'tiver sido pago': é algo como um futuro anterior. (Baudrillard, 1968: 219-220)

É neste sentido, por exemplo, que parece apontar o modelo de prospective accounting de Prelec e Loewenstein (1998), quando estes autores destacam o papel da ligação entre a dor do pagamento e o prazer do consumo (coupling), que se encontra enfraquecida numa compra a crédito. Apesar de podermos antecipar e usufruir do objeto antes de ele ser pago, i.e., de uma forma condicional, apenas consumamos a posse quando o consumo o transformou já em algo exaurido ou, pelo menos, diminuído no seu valor - adaptação hedónica que se posiciona hoje no centro da análise da psicologia hedónica e da economia comportamental do bem-estar (Kahneman et al., 1999; Hsee et al., 2008). A função social do crédito que não diabolizamos quando se considera o seu papel no 'projeto de vida', não deixa de conter um risco simultaneamente social, 
com efeitos de massa hoje sobejamente conhecidos, e individual, jogando explícita ou implicitamente com um "funcionamento" pessoal e familiar de "capacidade limitada" (Simon, 1990). Se comprar a crédito constitui um ato económico individual e/ou coletivo (família), ele realiza-se naquilo a que Baudrillard chama "desdobramento da compra nas suas determinações objetivas. Comprar a crédito equivale à apropriação total de um objeto por uma fração do seu valor real. Um investimento mínimo para um proveito grandioso." (1968: 225).

Distingue-se, por este mesmo facto, da aquisição direta de um bem ou serviço em que a utilidade daquilo que é comprado, e o seu preço, se apresentam simultaneamente ao juízo do consumidor que, decidindo sobre o seu justo balanço, escolhe comprar ou não comprar. Na verdade, o uso do crédito dificulta a representação desse equilíbrio, na medida em que o balanceamento entre benefício e custo apenas se constrói mediante a ponderação de um terceiro elemento - o tempo. Não queremos com isto sugerir que a dimensão temporal se encontra ausente numa decisão de compra, pois adquirimos sempre algo que vamos consumir ao longo do tempo (nem que seja já apenas na nossa memória) e gastamos sempre um montante financeiro do qual não vamos poder usufruir no futuro. Queremos antes sublinhar que o tempo adquire uma importância especial nas compras a crédito, uma vez que integra em si mesmo o verdadeiro custo da operação - o custo ligado ao objeto ou serviço, que não se esgota no valor de uma prestação, e o custo do seu usufruto antecipado, que corresponde ao preço do crédito.

Por agora, basta referir que, antes de efetuado o crédito, os processos cognitivos e afetivos envolvidos no julgamento e avaliação do(s) bem(ns) ou serviços de consumo coincidem em quase tudo com os de uma qualquer compra a pronto pagamento. Contudo, assim que o crédito ocorre como possibilidade real de financiamento, as avaliações de preço, utilidade, esforço e valor que determinariam numa compra instantânea a decisão final da aquisição, enquadram-se já num plano em que incerteza e risco emergem como elementos peculiares nem sempre devidamente avaliados. Decidir sobre o crédito implica uma decisão prévia sobre a compra, ou sobre aquilo que o dinheiro emprestado permite alcançar, e o julgamento de crédito não se separa nunca desta herança afetiva ligada à expectativa de obtenção de algo desejado ou necessitado. No entanto, a compra a crédito, na exata medida em que introduz este duplo momento decisional, distingue os processos de julgamento subjacentes a cada um deles. Numa primeira fase impera a força motivacional inerente à compra, e aqui os sinais positivos encontram poucas barreiras, 
uma vez que só numa segunda fase se avaliarão os seus custos. Neste subsequente passo, os custos do crédito não são nunca avaliados per si, mas sempre em concorrência com um outro custo - o de não efetuar a compra. É neste momento em que se avaliam as vantagens ou desvantagens do endividamento, e não em qualquer outro, que a decisão de crédito se reveste de real valor psicológico.

Deste ponto de vista, o recurso ao crédito coloca alguns desafios interessantes relacionados de forma direta com a própria estrutura intertemporal imposta pelo contrato de crédito que neste artigo procuramos identificar: pagar depois para ter antes o que de outro modo não se podia ter. Esta circunstância intertemporal particular coloca o consumidor no centro de inusitados conflitos, que emergem em grande parte à sua revelia (Botvinick et al., 2001). Os comportamentos daí resultantes, por vezes aparentemente irracionais, decorrem de formas de funcionamento mental e comportamental (automático-controlado, consciente-inconsciente, impulsivo-deliberado) que promovem a resolução dos conflitos por meio da operação de distintos sistemas de processamento de informação (dual system processing): um sumariamente denotado de intuitivo ou experiencial (Sistema I) e outro analítico ou racional (Sistema II) (Kahneman, 2003; Stanovich e West, 2000). Do ponto de vista funcional, estes dois sistemas apresentam características muito distintas. O Sistema I funciona num regime caracterizado por operações rápidas, automáticas, associativas, implícitas (ou impenetráveis à consciência), que exigem pouco esforço e são carregadas de tonalidade emocional. Já o Sistema II apresenta características funcionais que correspondem a operações lentas, seriais, que exigem esforço. São passíveis de monitorização consciente e alvo de deliberações (Smith e DeCoster, 2000; Strack e Deutsch, 2004). Os conteúdos de um e outro sistema, apesar de manterem relações funcionais entre si, são de natureza diferente, revertendo em consequências avaliáveis como muito diversas do ponto de vista do controlo consciente e, por isso mesmo, da liberdade e responsabilidade individuais.

É este processamento dual que hoje emerge como paradigma de explicação dos processos cognitivos/afetivos envolvidos na tomada de decisão (cf. St. Evans, 2008). Será por via destes dois sistemas, do seu funcionamento preponderante intricado, que o consumidor avalia o estabelecimento de um compromisso temporal com o crédito. Os resultados das decisões em consequências comportamentais abertas (e.g. contrair ou não o crédito, escolher um período de repagamento maior ou menor), assentam nesse funcionamento dual, que produz avaliações subjetivas (psicofísicas, afetivas) dos elementos concretos 
em presença (percebidos no momento ou recuperados da memória de longo prazo), insertos na condição intertemporal específica do crédito: o tempo presente. Tal significa calcular, comparar, categorizar, antecipar e prever consequências futuras diversas (e.g. valores, emoções) e, num regime consciente (ou não), cotejá-las com eventuais homólogos/ /análogos experienciais passados (ou a sua inexistência), sob a influência de elementos incidentais e integrais que se sabe hoje contribuírem diretamente na determinação das escolhas (pressão de tempo, Svenson e Maule, 1993; bumor incidental, Schwarz e Clore, 1983; fluência de processamento, Schwarz et al., 2009, entre outros).

\section{Escolher hoje resultados para amanhã - A arquitetura intertemporal do crédito}

Do ponto de vista clássico, a escolha intertemporal (Frederick et al., 2002) coloca a questão central do modo como se deve proceder à avaliação da utilidade de um objeto em diferentes intervalos de tempo futuro comparados com o tempo presente. No desconto intertemporal da utilidade, o modelo clássico do Desconto de Utilidade (DU) (Samuelson, 1937) é por vezes considerado como normativo e, portanto, referência racional (ver Frederick et al., 2002). Em termos gerais, para objetos de igual valor, a preferência é sempre dada ao objeto que se aloca primeiro no tempo em detrimento daquele que é diferido ou, numa linguagem comportamental, recompensas mais próximas no tempo, ainda que mais pequenas em valor, são preferidas a recompensas adiadas, maiores em valor. A utilidade futura é, assim, descontada a uma taxa que se mantém constante ao longo do período de tempo. Se o aumento em utilidade do objeto diferido no tempo não superar a taxa a que aquela é descontada, o objeto diferido no tempo continua a ser preterido face ao objeto alocado ao presente, mantendo-se esta preferência estável à medida que o tempo avança.

Diferentemente, o desconto intertemporal hiperbólico, resultante de testes empíricos do DU (Laibson, 1997; Frederick et al., 2002; Soman et al., 2005), envolve dois objetos de igual valor (ou o mesmo objeto) apreciados em momentos distintos com a preferência a recair, uma vez mais, naquele que se encontra primeiro na linha temporal. Contudo, a taxa a que tal desconto é operado não se mantém igual ao longo do tempo, sendo que, em intervalos de tempo considerados mais próximos do presente, a utilidade é descontada a uma taxa superior àquela que se verifica para intervalos mais afastados no tempo. À medida que se afasta do presente, o que o tempo lhe retira é cada vez menos. O desconto 
hiperbólico corresponde, assim, a uma avaliação no tempo presente de um tempo dele afastado, em que uma desvalorização em função do grau de afastamento se impõe, caracterizando-se por uma acentuada sensibilidade para com intervalos de tempo entre presente e momentos dele próximos, sensibilidade essa que diminui à medida que o intervalo representa maior afastamento.

O cálculo e estabelecimento das taxas de desconto, tal como resultam de dados obtidos em tarefas de escolha intertemporal, implicam que as pessoas façam comparações sequenciais das utilidades expressas por valores de indiferença entre um momento presente e diversos momentos futuros. Por exemplo, "quanto teriam de lhe pagar para aceitar não receber hoje um bem desejado (e.g. um telemóvel) e recebê-lo apenas daqui a uma semana?" (Thaler, 1980, 1985). Quer dizer, o valor é construído do ponto de vista da utilidade associada ao seu consumo. A evidência produzida aponta ainda para que a escolha intertemporal seja mais instável e dinâmica do que um simples desconto de utilidade futura faria prever - o fenómeno da instabilidade dinâmica. Com efeito, Strotz (1956) mostrou empiricamente que participantes numa experiência por si realizada preferiam, de acordo com o desconto intertemporal, "uma maçã" hoje a "duas maçãs" amanhã. Testados mais tarde sobre qual seria a sua preferência entre "uma maçã" daqui a "um ano" ou "duas maçãs" daqui a "um ano mais um dia" a escolha inverteu-se, agora em favor da segunda alternativa. As pessoas consideraram o mesmo intervalo de tempo ("um dia") no futuro (daqui a "um ano") menos importante do que "hoje", valendo a magnitude da segunda alternativa "duas maçãs" como determinante da inversão das preferências revelada.

A investigação em torno destas tarefas produziu evidência de que o valor para aceitar o adiamento de algo desejado é muito elevado quando comparado com o valor nominal atual do bem, significando que o sujeito desconta consideravelmente o valor do bem no futuro (e.g. uma semana), embora este permaneça teoricamente inalterado. $\mathrm{Na}$ verdade esta expressão de valoração do presente corresponde a um enviesamento do julgamento de utilidade da decisão para o momento presente - present bias. Esta comparação de valores de bens (ou o que quer que seja) apreciados num futuro mais ou menos distante ao presente (bic et nunc) implica uma dupla presentificação: a avaliação do presente e a avaliação do futuro à luz do presente.

\subsection{O crédito como resultado de uma preferência intertemporal}

Do ponto de vista comportamental e mental, o crédito apresenta um carácter especial pela relação intertemporal que faz instalar na sua circunstância: 
o modo como ganhos imediatos têm preferência relativamente a ganhos diferidos no tempo e como, no sentido oposto, as perdas adiadas são preferidas às imediatas (Ranyard e Craig, 1995). Concretizando os modelos de utilidade descontada e partindo, como nesta nossa análise é pressuposto, de que a decisão de compra está tomada, a escolha entre uma recompensa maior adiada e uma recompensa menor mais cedo é análoga a uma escolha entre obter o bem ou serviço através de poupança ou através de crédito. Fazer uma poupança equivale a ter uma recompensa maior na medida em que o custo do produto não sofre um acréscimo de valor, pois apenas é adquirido no futuro. Por outro lado, fazer um crédito permite ter agora (mais cedo) mas, pagando mais por isso (juro), faz com que o valor final da recompensa seja menor, porque mais caro. Comportando-se a avaliação das punições de forma simétrica, sabemos também que as punições no futuro são percebidas como tendo menos desutilidade do que punições mais próximas do presente, levando a que punições maiores mais tarde sejam preferidas a punições menores mais cedo, ou seja, a que seja preferível diferir o pagamento a pagar no imediato.

Para além disso, as punições associadas ao crédito surgem apresentadas num formato que favorece a perceção de uma magnitude reduzida: o benefício da compra é comparado com o pagamento de uma prestação mensal, que apenas reflete parte do valor do crédito.

Começa assim a construir-se a ideia de que o crédito, como produto financeiro arquitetado, encaixa neste modus de funcionamento humano. A sua estrutura intertemporal operacionaliza um raciocínio de valorização do presente e coloca o decisor num vasto espaço de incerteza onde outras tantas formas de raciocínio entram em jogo, podendo claramente criar contextos de vulnerabilidade.

\subsection{As probabilidades são subjetivamente construídas. Crédito e efeito da pseudo-certeza}

Keren e Roelofsma (1995) levaram a cabo experiências que corroboram a existência de uma relação entre, por um lado, imediação e certeza e, por outro, adiamento e incerteza. A avaliação em separado de prospetos imediatos gera uma perceção de certeza associada aos mesmos (probabilidade igual a 1), enquanto os prospetos adiados são percebidos como incertos (Weber e Chapman, 2005). Esta relação imediação/ /certeza e adiamento/incerteza parece conduzir a um efeito clássico de distorção percetiva da probabilidade, obtido anteriormente por Tversky e Kahneman (1981) e conhecido por efeito de pseudo-certeza. Este efeito ocorre quando, num jogo de duas fases apresentadas simultaneamente, 
a escolha dos jogadores difere daquela que é feita quando esse mesmo jogo é apresentado como tendo uma só fase, violando o princípio da invariância (von Neumann e Morgenstern, 1944).

Seguindo um desenho experimental emprestado daquele que Tversky e Kahneman (1981) usaram para ilustrar o efeito de pseudo-certeza, Li, Su e Sun (2010) demonstraram existir também um efeito de pseudo-imediação. Por outras palavras, risco e adiamento parecem ser psicologicamente equivalentes, tal como imediação e certeza (Li et al., 2010), conduzindo a perceções distorcidas da relação das probabilidades (pseudo-certeza) e da relação intertemporal imediato/diferido (pseudo-imediação). Em ambos os casos ocorre sobrestimação de pequenas probabilidades ou de imediação de recompensa com base num elemento de certeza/imediação condicionado por uma probabilidade ou período de acesso prévios que acabam negligenciados. Imagine-se a situação de um consumidor que, uma vez decidido a adquirir um bem para o qual não tem rendimento disponível, recorre a um crédito. Esta situação de consumo bastante comum é análoga à situação do jogo em que para aceder a uma segunda fase, no caso obter o usufruto imediato/certo (crédito) ${ }^{1}$ ou diferido/incerto (poupança) do bem, terá de considerar numa primeira fase as incertezas que cada uma das alternativas impõe, ${ }^{2}$ ou seja, as possibilidades de cumprimento que as condições contratuais do crédito e de aforro implicam. Esta primeira fase apresenta pois um risco que o consumidor terá de formular repartindo probabilidades de cumprimento e incumprimento de ambos. Donde o acesso à segunda fase (aquisição) estaria sempre condicionado ao balanço dessas probabilidades e não à expectativa do usufruto ou não-usufruto imediato do bem. $\mathrm{O}$ adiamento do usufruto que a poupança implica resulta numa perceção de incerteza (relação adiamento/incerteza) que comparada ao usufruto imediato consentido pelo crédito conduz a uma perceção de certeza (relação imediação/certeza). Tais relações centram a perceção no carácter imediato/certo do usufruto em detrimento da possibilidade de poupança, o que deverá conduzir à negligência dos condicionantes que a primeira fase impõe (ponderação cumprimento/ /incumprimento e aforro).

A partir desta estrutura ou arquitetura da decisão intertemporal inerente ao crédito, podem traçar-se linhas de resposta psicológica que

\footnotetext{
${ }^{1}$ Sublinhamos a ideia de que o usufruto não se confunde com posse.

2 Por exemplo, instabilidade do emprego, saúde, entre outras que se sabe determinarem situações de incumprimento ou dificultarem o vencimento de um plano de poupança.
} 
constituem vias de vulnerabilização do julgamento e tomada de decisão do consumidor no que toca, especificamente, à antecipação das consequências incertas associadas ao crédito. Este, enquanto garantia de aquisição que de outro modo não seria possível ou desejável, transforma a probabilidade de aquisição numa certeza $(100 \%)$ e reduz a uma impossibilidade $(0 \%)$ outras alternativas de aquisição: poupança ou simples diferimento para contrair crédito em condições mais favoráveis. E mais ainda: parece favorecer a ocultação de riscos inerentes precursores do incumprimento, uma vez que o adiamento pode ser equacionado como incerteza e, consequentemente, afastado de deliberação (efeito de pseudo-imediação).

\section{Decidindo fazer o crédito 3 - Processos cognitivos/afetivos de julga- mento e decisão}

A este condicionalismo estrutural imposto do exterior ao sujeito, e que emana unilateralmente do ordenamento jurídico, económico e social que enquadra o crédito, junta-se um outro de natureza interna, decorrente de um funcionamento psíquico complexo e, fundamentalmente, adaptativo. Os elementos situacionais da estrutura intertemporal do crédito para um sujeito são processados enquanto estímulos hic et nunc por um aparelho cuja constituição e funcionamento dá origem a comportamentos com carácter indeterminado e instável. Propomos agora, percorrendo breve e seletivamente alguns dos dados atuais da psicologia do julgamento e tomada de decisão, em especial em torno do eixo das relações entre cognição e emoção, fazer salientar as indeterminações decorrentes da complexidade do processamento dual envolvido na produção de julgamentos e decisões, ativados perante a avaliação de compromissos exigidos pelo crédito. O propósito fundamental da discussão que se segue é o de mostrar como a complexidade de que se reveste este jogo, responsável pela instabilidade e indeterminação inerentes ao comportamento humano, se exprimem em particular no que toca à situação típica do crédito.

\footnotetext{
${ }^{3}$ Não consideramos aqui os processos concretos que conduzem à decisão de fazer uma compra. Contemplamos antes como os produtos dessa decisão, que emergem agora como entradas no sistema de decisão de contrair um crédito, o condicionam. Focamos pois o momento em que o presente da decisão de tomar ou não o crédito pode constituir um cadinho de vulnerabilidades individuais (que podem ser também coletivas) passíveis de manipulações várias. Para uma descrição genérica de como este processo pré-decisão do crédito, bem como o que resulta num período pós-decisional, podem ser descritos (ver Kamleitner e Kirchler, 2007).
} 


\subsection{Sistemas cognitivos de capacidade limitada: impacto na qualidade de pro- cessamento de informação e na autorregulação do comportamento}

Desde logo, a quantidade e a complexidade da informação (Sweller, 1988), a taxa e a densidade temporal de execução (Barrouillet et al., 2004), bem como a ordem com que nos chega, têm um impacto sobre o desempenho dos sistemas de tratamento de informação de capacidade limitada ${ }^{4}$ - como é caso da memória de trabalho (Baddeley, 1986; 2000) - determinando em grande parte o modo como decidimos. É um dado claro, do ponto de vista teórico como empírico, que a quantidade de informação coloca uma pressão funcional no seu processamento (Posner, 1993; Simon, 1957; Styles, 2005: cap. 12). Esta pressão determina que o desempenho na realização de duas tarefas em simultâneo (dual-task) fique seriamente comprometido quando comparado com o desempenho de cada uma delas em separado (Gathercole, 2008; Kahneman, 1973). A fragilidade intrínseca do funcionamento destes sistemas tem como consequência, por exemplo, que a mera evocação (recall) de conteúdos elementares possa ser crucialmente prejudicada pela recuperação de conteúdos simples da memória de longo prazo (Rohrer et al., 1998).

Imposta por evocação de sentimentos sobre o tempo disponível, uma condição contextual crónica do funcionamento nas nossas sociedades atuais (Szollos, 2009), a pressão de tempo é responsável pela alternância entre regimes de processamento de informação, uma vez que a menos tempo disponível corresponde maior complexidade da tarefa (Payne et al., 1993; Oliveira, 2005). O seu efeito mais reconhecido é o do "estreitamento do foco de atenção" (cf. Hammond, 2000; Wright, 1974; para outros efeitos ver Edland e Svenson, 1993; Maule e Edland, 1997; Maule et al., 2000; Payne et al., 1993) e no consumo parece favorecer a redução do adiamento da aquisição quando existe um conflito patente entre alternativas (e.g. igual atratividade das alternativas), efeito que tende a extinguir-se quando não existe pressão de tempo (Dhar e Nowlis, 1999).

Todos estes efeitos, que alteram a carga cognitiva, impõem um funcionamento mais instável dos sistemas e podem conduzir a resultados que exprimem fraco controlo atencional no que toca a conteúdos cognitivos básicos e de alto nível, como são as metas e motivos que servem de referência autorregulatória na decisão (Hofmann et al., 2012). Um bom exemplo do efeito de carga cognitiva (ou de capacidade cognitiva diminuída) sobre

\footnotetext{
${ }^{4}$ Integram a cognição consciente, atenção seletiva, memória imediata e o controlo voluntário, a par dos processos de grande capacidade: memória de longo prazo, competências ou habilidades altamente aprendidas e treinadas (rotinas, hábitos) e vocabulário (Baars, 2007).
} 
processos autorregulatórios é dado por Friese et al. (2008) num estudo realizado com medidas de atitudes explícitas e implícitas face a objetos de consumo (peças de fruta e barras de chocolate), que demonstravam ser antagónicas (caso de participantes que sustentavam simultaneamente preferência explícita por fruta e preferência implícita por chocolate). Os participantes tinham de escolher retirando peças de fruta ou barras de chocolate de uma taça enquanto mantinham na memória oito dígitos (capacidade cognitiva diminuída) ou um dígito (capacidade cognitiva elevada). Mostrou-se que o conflito de avaliação entre fruta e chocolate não pode ser resolvido de acordo com as preferências explícitas por fruta previamente medidas (produzidas pelo Sistema II, analítico) quando a memória de trabalho está mobilizada pela manutenção de oito dígitos, fazendo convergir as escolhas para preferências implícitas por chocolate (produzidas pelo Sistema I, experiencial), denotando uma falha de autocontrolo, que não ocorre quando apenas um dígito tem de ser mantido na memória. Extrapolando para situações análogas relativas ao crédito, as pessoas que explícita e deliberadamente defendam atitudes em seu desfavor podem acabar por revelar preferências inversas em situações de carga cognitiva elevada, em convergência com fortes motivos ou necessidades implícitas de aquisição de bens ou serviços.

Embora as pessoas consigam frequentemente influenciar os seus próprios pensamentos, sentimentos e motivos de acordo com prioridades representadas conscientemente, este normal funcionamento do controlo mental parece apenas ter sucesso quando há capacidade para o seu exercício (Wegner, 1994). Em caso de diminuição dessa capacidade, a falha do sistema pode resultar tanto da inexistência de controlo (como no exemplo anterior) como da criação de estados mentais opostos àqueles que são desejados, aquilo a que Wegner $(1994,1997)$ chamou efeitos irónicos do controlo (EIC). Num caso hipotético de controlo do desejo de fazer umas férias pagas a crédito, os recursos mentais (atenção) são alocados em imagens relacionadas com férias (e.g. praia, viagens), que sinalizam, para efeitos de controlo, o que se pretende evitar. Ironicamente, este processo favorece o acesso privilegiado dessas imagens à consciência, conduzindo a um comportamento consistente com o sentimento que carregam: realizar as férias a crédito.

Também a depleção do ego, uma condição de autorregulação diminuída, foi estudada por Baumeister et al. (2008) e consiste na redução da capacidade de autorregulação resultante do seu exercício prévio, com impacto em comportamentos subsequentes, ligados ou não à situação de exercício do controlo. É a situação de um consumidor que 
após realizar as compras com o auxílio de uma lista, encontrando-se satisfeito por ter resistido às compras excessivas (só comprou os itens listados), resolve confirmar o preço de um LCD que há muito deseja e acaba por adquiri-lo a crédito, embora nutrisse reservas consistentes e elaboradas contrárias a esse tipo de financiamento. Alguns estudos sugerem que o estado de depleção de recursos de autocontrolo do ego corresponde a um funcionamento típico do Sistema I (Hamilton et al., 2007; Wang et al., 2010).

\subsection{Afeto como informação - Experiências subjetivas de acessibilidade e fluência de informação}

Para além dos efeitos com impacto no processamento de informação é importante salientar que os pensamentos são ainda acompanhados de experiências subjetivas (Schwarz, 2004) na forma de estados de humor, emoções, experiências metacognitivas e sensações corporais (Schwarz, 2012). Não faltam dados sobre estas experiências subjetivas, produzidos no âmbito do paradigma de sentimentos como informação (Schwarz e Clore, 1983; Schwarz, 2012). Neste, assume-se que os sentimentos veiculam informação que por sua vez influencia a formação de julgamentos usados nos processos de tomada de decisão. Estas avaliações subjetivas não recaem sobre as informações (e.g. números, palavras, imagens, metas), mas comunicam a facilidade ou dificuldade com que se lhes acede e a fluência com que é processada (Schwarz et al., 2007). Seria o caso de um consumidor a quem se pede para elencar mais de três ou quatro "razões para um possível incumprimento" num crédito: desemprego, divórcio, baixa de salário, doença, etc. Embora não relacionado com estas, o sentimento de dificuldade associada à geração das razões é uma informação (negativa neste exemplo) que indica, todavia, não existência de muitas mais "razões" ou a pouca probabilidade de existirem. O sentido desta informação (valência) contraria o conteúdo da informação declarativa das "razões de incumprimento", levando à desvalorização do seu conteúdo negativo. Já a geração de "razões para o sucesso" do crédito (agora positivas) poderá tornar-se uma tarefa fácil, já que o sujeito delibera com base na decisão de aquisição prévia, imaginando-se na posse do bem, favorecendo desse modo o acesso a informação positiva.

Para além da facilidade ou dificuldade de acesso à informação, também o sentimento de facilidade ou dificuldade na perceção direta dos estímulos (fluência) pode condicionar julgamentos e decisões. Experiências realizadas no âmbito do paradigma de sentimentos como informação (Schwarz, 2012) 
mostram que, por exemplo, a informação mais fácil de processar ${ }^{5}$ é avaliada como familiar e por isso percebida como indicando menos risco, tal como a informação difícil de processar sinaliza um risco maior (Song e Schwarz, 2009).

Os informadores financeiros típicos das ofertas de crédito ao consumo, expressos em unidades que os sujeitos conhecem e escalas com que lidam no seu quotidiano (familiares), como é o caso de euros e/ou meses (prestação do montante total imputado ao consumidor [MTIC] e duração), são facilmente posicionáveis numa dimensão de bom/mau, alto/baixo, suportável/não suportável. Já os elementos referentes às taxas poderão ser de difícil processamento. A TAN ou a TAEG obrigam a fazer "contas" para perceber quantos euros representa, por mês ou durante toda a vigência do crédito, uma determinada taxa. Um aumento de TAEG pode ser percebido como mais arriscado do que um aumento de prestação ou duração. Uma vez que montantes de reembolso maiores e prazos de reembolso menos alargados são também os que apresentam TAN e TAEG mais elevadas, a tentativa de evitar o risco de uma taxa elevada poderá levar o consumidor a optar por um crédito que o compromete durante muito tempo, expondo-o às conhecidas dificuldades de previsão, aumentando a sua incerteza e pondo em causa, no limite, o cumprimento do contrato, para além deste tipo de contratos originar MTICs superiores. Por outras palavras, TAN e TAEG tenderão a transmitir ainda maior risco devido à sua difícil fluência percetiva.

\subsection{Afeto na avaliação de acontecimentos futuros}

Os nossos afetos estão não só ligados à forma como geramos e percebemos a informação (e.g. fluência) relativa ao presente ou ao futuro, mas também à forma como antecipamos no presente os nossos estados afetivos futuros. É interessante referir que comparada com a recuperação de imagens e sentimentos passados, a geração de imagens e sentimentos futuros parece acompanhar-se de maior intensidade emocional (Van Boven e Ashworth, 2007).

Um dos mais robustos e prevalentes efeitos de distorção reportados em estudos sobre bem-estar e felicidade (Frey, 2008) é o da adaptação hedónica (Frederick e Loewenstein, 1999) ou a condição em que eventos afetivamente bons ou maus só temporariamente influenciam o bem-estar autorrelatado (ver Diener et al., 2006). As pessoas detêm uma capacidade

\footnotetext{
5 Por exemplo, similaridade semântica ou frequência e recência da exposição (Schwarz et al., 2007).
} 
para voltar rapidamente a um estado de relativa neutralidade hedónica (hedonic treadmill) e é a negligência destas capacidades de regulação emocional (Gross, 2007; Vohs e Baumeister, 2011) que resulta por vezes em previsões descalibradas (Wilson e Gilbert, 2005).

$\mathrm{Na}$ previsão de acontecimentos futuros pontuam outros fenómenos de distorção preditiva, como é o caso do "fosso empático" (empathy gap), descrito e proposto por Loewenstein e Schkade (1999). Neste, é a discrepância entre o contexto emocional/afetivo da previsão e o contexto em que o acontecimento é efetivamente experienciado que determina a distorção: num contexto de previsão visceralmente "frio" (ativação e intensidade emocional baixas) (Loewenstein, 1996), torna-se difícil imaginar e prever o estado afetivo ou emocional futuro relativo a um dado acontecimento focal em que o contexto passa a ser visceralmente "quente" (e.g. fome, stress, dor). Por exemplo, a perceção e avaliação do esforço de pagamento da prestação mensal como tolerável num contexto "frio" pode previsivelmente alterar-se quando o contexto se torna "quente", devido à constatação de uma despesa extra inesperada decorrente de um problema de saúde.

Independentemente da carga visceral do contexto, também a previsão de gostos (e.g. previsão da saciação de um alimento) sofre distorções notórias. Em estudos hoje clássicos, Kahneman e Snell $(1990,1992)$ verificaram que, aparentemente, as pessoas parecem conhecer com relativa precisão os seus gostos, mas conhecem mal o modo como sobre eles se vão sentir no futuro: as previsões de alteração do prazer sentido apresentam correlações ínfimas com as alterações efetivamente sentidas (Kahneman e Snell, 1992).

Fenómenos de distorção percetiva da previsão agregados em torno do focalismo (Wilson et al., 2000; Gilbert e Wilson, 2011), ou seja, da centração num único evento futuro agradável/desagradável (e.g. adquirir automóvel ou ir de férias/pagamento do crédito), geram avaliações afetivas implícitas ou explícitas associadas, cuja projeção no futuro parece conduzir a distorções de avaliação do seu impacto e duração (Wilson e Gilbert, 2003; 2005). Na avaliação do impacto, o focalismo conduz a atribuição de maior importância ao afeto associado ao evento do que aquela que em momentos futuros concretos as pessoas usualmente lhe concedem. Tal importância diminui significativamente quando o evento é avaliado num contexto mais alargado e plausível, no qual outros eventos associados (avaliados positiva ou negativamente) são agora considerados, mitigando o seu impacto. Já a durabilidade do impacto parece resultar de outros mecanismos. Existe evidência de que a previsão de quanto dura 
o impacto de eventos positivos ou negativos é alvo de distorções que decorrem daquilo que Gilbert et al. (1998) apelidaram de negligência da imunidade psicológica (immune neglect), ou seja, o desprezo do nosso potencial para lidar com situações negativas.

Tomemos o caso em que a perspetiva da compra de um automóvel é negativa devido ao preço elevado, inviabilizando-a. Prevendo um impacto negativo de não ter o carro nos tempos mais próximos, quanto tempo se prevê que vai durar tal sentimento? A distorção (durability bias) ocorre se o sentimento negativo for avaliado com uma intensidade constante ao longo desse período, o que estudos de follow-up (Gilbert et al., 1998) demonstraram realmente não acontecer. E no caso de a antecipação ocorrer relativamente à aquisição efetiva do carro a crédito? A antecipação seria agora de sentido positivo, uma vez que o crédito oferece a possibilidade de consumar o desejo. Tal sentimento poderá levar à sobrestimação da sua durabilidade, um fenómeno bem conhecido na antecipação do consumo (Alba e Williams, 2013; Wilson et al., 2005).

\subsection{Modelos integradores}

Constrangimentos de espaço obrigam-nos a referir apenas de forma muito sucinta duas propostas teóricas que prometem explicações mais integradoras de como o processamento dual de informação (acima descrito) ou o processamento de informação suscitado pela dimensão distância (em especial, a temporal) associada à arquitetura do crédito determinam julgamentos e decisões. Uma primeira, a Heurística Afetiva (Slovic et al., 2007), pretende explicar os nossos julgamentos e decisões com base na confiança que tendemos a depositar em avaliações afetivas dos estímulos integrantes dos contextos de decisão. Rápidas - fruto do funcionamento típico do Sistema I, resultando naquilo que Oliveira (2007) apelidou de "hedonização da cognição" - e decorrentes da nossa experiência privada, tais avaliações gozam de um poder que lhes advém da simplificação que promovem (heurísticas) e do significado que conferem a situações complexas e exigentes para o processamento de informação. Slovic et al. (2007) mostram, invocando diversas linhas de investigação, que os julgamentos e decisões construídas com base em avaliações afetivas são modulados por diversas incidências contextuais por nós aqui já referidas (e.g. pressão de tempo). Problematizam os seus efeitos como fontes de vulnerabilidade na construção de julgamentos devido ao correspondente potencial de manipulação (ver em especial, no crédito, Estelami, 2001). É neste sentido que acreditamos que a publicidade e o marketing funcionam como formas de exploração da arquitetura de crédito, intensificando 
a sua estrutura rígida. Esta potencia um funcionamento psicológico que reforça avaliações afetivas associadas ao consumo e favorece a eficiência na decisão de crédito, relegando para um plano secundário deliberações de outras alternativas.

Já a proposta da Teoria dos Níveis de Construto (Trope e Liberman, 2010) afirma que a distância objetiva (temporal, ao eu, social, de hipoteticalidade) é psicologicamente construída, invocando diferentes representações mentais resultantes de graus distintos de processamento de informação (alto/baixo nível) e, consequentemente, determinando a natureza dos julgamentos e decisões. A hipótese central é a de que, quanto mais afastado do tempo presente (ou nas outras dimensões) se encontra o alvo da elaboração psicológica (e.g. aquisição de um bem), mais esta tenderá a mobilizar um processamento de alto nível, resultando em julgamentos mais abstratos (e.g. comprar roupa de marca é bom porque me aproximo de um ideal de pertença social). Neste caso, a construção psicológica dos julgamentos de antecipação não depende do detalhe e concretude próprios dos contextos imediatos da experiência. Estes últimos decorrem de um processamento dos estímulos e seus atributos (e.g. a cor de uma camisola ou da decoração da loja) a um baixo nível (sensório-motor e percetivo), suscitados por uma distância temporal mais próxima. O peso da informação processada a partir dos estímulos e seus atributos percebidos diretamente para distâncias temporais mais curtas diminui com distâncias crescentes, aumentando concomitantemente o peso de informação processada a alto nível. Esta proposta parece-nos ser interessante para iniciar o estudo das representações dos informadores típicos do crédito, em particular o caso das prestações. Será interessante verificar como evolui a sua representação, dada a óbvia inserção num conjunto de horizontes temporais crescentes em distância ao presente. Existirá um ponto de corte temporal a partir do qual o processamento dos valores das prestações deixa de ser preponderantemente de baixo nível e, portanto, avaliado em termos de exequibilidade e cumprimento do empréstimo (e.g. o rendimento disponível) e passa, pela distância maior ao presente, a ser de alto nível associando-se agora a elementos de desejabilidade do objeto que motiva o crédito (ver Liberman, Trope e Waslak, 2007)?

\section{Notas finais}

Quando nos referimos à arquitetura da decisão de crédito queremos indicar o desenho da articulação dos elementos básicos que constituem a situação efetiva de tomar hoje posse daquilo que hoje não se poderia objetivamente adquirir com base no rendimento disponível, adiando o 
pagamento para um futuro mais ou menos extenso e incerto, apesar de formalmente repartido e referenciado em horizontes temporais especificados (prestações).

Sem qualquer preocupação de avaliar de acordo com diferentes perspetivas de racionalidade (Gigerenzer, 2010), o ponto aqui é simplesmente este: os elementos constituintes implicados na arquitetura do crédito parecem ser fonte de um funcionamento psicológico/comportamental muito variado e instável, quando adotada a perspetiva da racionalidade limitada. Dos processos cognitivos realizados em regimes de capacidade limitada aos processos de avaliação e previsão afetiva por nós referidos, o único padrão parece ser o de uma construção dependente do contexto imanente a todo o processo de decisão, o que é aliás consistente com a designação sintética de Lichtenstein e Slovic (2006) de construção das preferências, ou das atitudes (Schwarz, 2007). Corresponde assim a uma massa complexa de contribuições várias que constituem os processos cognitivos/ /afetivos que procurámos aqui sinalizar em torno da arquitetura do crédito. Tal exercício não autoriza, porém, a muito desejada capacidade de previsão, que é, explícita e implicitamente, comummente exigida à ciência.

Procurámos mostrar que à complexidade e incerteza de que se revestem em geral os processos de decisão intertemporal, o crédito impõe uma rigidez estrutural no comprometimento que contratualiza: troca-se a incerteza dos estados futuros avaliados de forma imprecisa pela certeza crua de pagamentos futuros. ${ }^{6}$ Se a maioria dos empréstimos são casos de "sucesso" em que os fatores de complexidade e incerteza se fazem sentir de forma que permita ajustamentos (bem entendido, por vezes conseguidos à custa de sacrifícios de outros consumos importantes), uma falha de ajustamento no cumprimento do contrato pode corresponder a desfechos nefastos e mesmo fatais. E isto quer no que respeita ao motivo que levou ao pedido de empréstimo (perda do bem/ serviço adquirido), quer no que respeita à perda de condições de vida já alcançadas, hipotecadas agora ao cumprimento crescentemente exigente que a sua liquidação implica. ${ }^{7}$

\footnotetext{
${ }^{6}$ Uma troca fundamental, que corresponde à ideia de que a escolha intertemporal desencadeia uma fragilidade significativa da vontade individual (Ainslie, 2001). A esta, outra fragilidade se associa: a impossibilidade de fracassar individualmente o que está bem para lá da mera vontade ou controlo individuais.

7 Desfechos nefastos tanto individuais quanto agregados, como a crise atual, infelizmente nos mostram por meio de um exército de sobreendividados sofrendo de um endémico "consumo da bolsa", na expressão de Falstaff do Henrique IV: "I can get no remedy against this consumption of the purse: borrowing only lingers and lingers it out, but the disease is incurable" (Shakespeare, 2013).
} 
Mais a mais, apesar de poderem planear e simular a implementação das suas intenções, não se encontram os sujeitos sempre e exatamente perante as mesmas complexidades objetivas desta arquitetura? À semelhança do que acontece com os participantes nas experiências de Tversky e Kahneman (1981) e de Li, Su e Sun (2010), não podem os contraentes de crédito incorrer numa inversão de preferências pela centração num elemento de probabilidade/imediação pouco transparente, resultante da arquitetura de decisão imposta pelo crédito?

Das distorções psicofísicas sobre probabilidades sinalizadas na Teoria dos Prospetos (Kahneman e Tversky, 1979), que explicam a pseudo-certeza em jogos como o que daí tomámos emprestado, às distorções afetivas da previsão (Kahneman e Snell, 1992; Gilbert e Wilson, 2011) que explicam o desacerto entre o impacto afetivo e duração previstos de algo desejável ou indesejável e a sua efetiva experiência, a construção das preferências e as decisões nela baseada sugerem que o problema que se coloca à função das entidades reguladoras implica uma visão e processos ainda mais difíceis de implementar do que à primeira vista poderia parecer.

Não se poderá certamente partir para uma regulação meramente reativa e redutora. Um exemplo curioso desta atitude e uma das ideias defendidas nestes tempos de crise por entidades reguladoras (e.g. Banco de Portugal, 2011) é a de que a numeracia e literacia financeiras permitiriam ultrapassar e impedir os "erros" de decisão devidos à complexidade inerente ao cálculo e planeamento de decisões financeiras (Huston, 2010; cf. Willis, 2008 para uma posição contrária). Porém, tais especulações sobre o valor da literacia financeira parecem descurar a mais básica consideração da "instabilidade natural" patente no padrão de funcionamento psicológico e a própria complexidade inerente aos produtos financeiros:

Do ponto de vista da proteção do consumidor, uma preocupação particular tem sido o agudo crescimento da complexidade dos produtos financeiros oferecidos aos consumidores, complexidade que tem sido um efeito secundário da inovação mas que também tem sido associada, em muitos casos, com a transparência e clareza reduzidas nos produtos oferecidos. (Bernanke, 2009: 2)

Sugestões "paternalistas/libertárias" como as de Thaler e Sunstein (2008) para melhorar a vida das pessoas passam por conceber intervenções (do Estado, mas também de organizações privadas da sociedade civil) por meio de "pequenos empurrões" ou "jeitos" (nudges) que, sendo casuísticos, dependem de cuidadosas análises e avaliações da arquitetura de escolha específica com que as pessoas se podem deparar. Detetados e 
assinalados esses riscos, difíceis de desenviesar ou anular, podem ser removidos e/ou devidamente sinalizados, garantindo, apesar dessa intervenção (paternalista), a liberdade individual de escolha (libertária). Outras, como a de Hogarth (2010), parecem exigir uma perspetiva que suscita a intervenção mais técnica e científica. Pessoas de diferentes condições sociais e económicas podem assim ser testadas, estudando-se em que medida as suas previsões e escolhas variam sistematicamente com as variações dos elementos arquiteturais da decisão.

Se à complexidade de contexto e de processo na tomada de decisão face ao crédito não pode corresponder uma previsão científica precisa de comportamentos, não é menos verdade que é possível traçar algumas linhas de atuação. Se se quer minimizar efeitos nefastos do endividamento, afigura-se-nos importante sugerir políticas de regulação em que a apresentação de propostas de crédito conjugue três elementos genéricos: explicitação de informação, consciencialização das consequências negativas do crédito e aperfeiçoamento da perceção da estrutura intertemporal do crédito. Estamos conscientes de que estes elementos carecem, ainda, de suporte científico empírico no sentido de promover a sua validade.

\section{Referências bibliográficas}

Ainslie, George (2001), Breakdown of Will. Cambridge: Cambridge University Press [2. ${ }^{a}$ ed.].

Alba, Joseph; Williams, Elanor (2013), "Pleasure Principles: A Review of Research on Hedonic Consumption”, Journal of Consumer Psychology, 23(1), 2-18.

Baars, Bernanrd J. (2007), "A Framework", in Bernard J Baars e Nicolas M. Gage (orgs.), Cognition, Brain, and Consciousness. London: Academic Press, 31-57.

Baddeley, Alan (1986), Working Memory. Oxford: Clarendon Press.

Baddeley, Alan (2000), "Episodic Buffer: A New Component of Working Memory?", Current Trends in Cognitive Science, 4, 417-422.

Banco de Portugal; Comissão de Mercados e Valores Mobiliários; Instituto de Seguros de Portugal (orgs.) (2011), Plano Nacional de Formação Financeira. 2011-2015. Lisboa.

Barrouillet, Pierre; Bernardin, Sophie; Camos, Valérie (2004), "Time Constraints and Resource Sharing in Adults' Working Memory Spans”, Journal of Experimental Psychology, 133(1), 83-100.

Baudrillard, Jean (1968), Le système des objets. Paris: Gallimard.

Baumeister, Roy; Sparks, Erin; Stillman, Tyler; Vohs, Kathleen D. (2008), "Free Will in Consumer Behavior: Self-control, Ego Depletion, and Choice", Journal of Consumer Psychology, 18(1), 4-13. 
Bernanke, Ben (2009), "Financial Innovation and Consumer Protection", discurso proferido na Federal Reserve System's Sixth Biennial Community Affairs Research Conference, 17 de abril, Washington, D.C. Página consultada a 07.05.2013, em http://www.federalreserve.gov/newsevents/speech/bernanke20090417a.htm?ref=nf.

Botvinick, Matthew; Braver, Todd; Barch, Deanna; Carter, Cameron; Cohen, Jonathan (2001), "Conflict Monitoring and Cognitive Control", Psychological Review, 108(3), 624-652.

Dhar, Ravi; Nowlis, Stephen (1999), "The Effect of Time Pressure on Consumer Choice Deferral", Journal of Consumer Research, 25, 369-384.

Diener, Ed; Lucas, Richard; Scollon, Christie (2006), "Beyond the Hedonic Treadmill: Revising the Adaptation Theory of Well-being”, American Psychologist, 61(4), 305-314.

Edland, Anne; Svenson, Ola (1993), "Judgment and Decision Making under Time Pressure: Studies and Findings”, in Ola Svenson; John Maule (orgs.), Time Pressure and Stress in Human Judgment and Decision Making. New York: Plenum Press, $27-40$

Estelami, H. (2001), "Determinants of Discount Rates in Consumer Credit Decisions", Journal of Marketing Theory and Practice, 9, 63-73.

Frederick, Shane; Loewenstein, George; O’donoghue, Ted (2002), “Time discounting and time preference: A critical review”, Journal of Economic Literature, 40(2), 351-401.

Fredrick, Shane; Loewenstein, George (1999), "Hedonic Adaptation”, in Daniel Kahneman; Ed Diener; Norbert Schwarz (orgs.), Well-being: The Foundations of Hedonic Psychology. New York: Russel Sage Fundation, 302-329.

Frey, Bruno (2008), Happiness. A Revolution in Economics. Cambridge, MA: The MIT Press.

Friese, Malte; Hofmann, Wilhelm; Wänke, Michaela (2008), "When Impulses Take Over: Moderated Predictive Validity of Explicit and Implicit Attitude Measures in Predicting Food Choice and Consumption Behavior”, British Journal of Social Psychology, 47, 397-419.

Gathercole, Susan (2008), "Working Memory”, in John Byrne (org.), Concise Learning and Memory. Amsterdam: Academic Press, 149-167.

Gigerenzer, Gerd (2010), Rationality for Mortals: How People Cope with Uncertainty. New York: Oxford University Press.

Gilbert, Daniel; Pinel, Elizabeth; Wilson, Timothy; Blumberg, Stephen; Wheatley, Thalia (1998), "Immune Neglect: A Source of Durability Bias in Affective Forecasting”, Journal of Personality and Social Psychology, 75(3), 617-638.

Gilbert, Daniel; Wilson, Timothy (2011), "Previews, Premotions and Predictions", in Moshe Bar (org.), Predictions in the Brain: Using our Past to Generate the Future. New York: Oxford University Press, 159-169. 
Gross, James (org.) (2007), Handbook of Emotion Regulation. New York: Guilford Press.

Hamilton, Ryan; Hong, Jiewen; Chernev, Alexander (2007), "Perceptual Focus Effects in Choice”, Journal of Consumer Research, 34, 187-199.

Hammond, Kenneth (2000), Judgments under Stress. New York: Oxford University Press.

Hofmann, Wilhelm; Schmeichel, Brandon; Baddeley, Alan (2012), "Executive Functions and Self-Regulation”, Trends in Cognitive Sciences, 16(3), 137-188.

Hogarth, Robin (2010), "Subways, Coconuts, and Foggy Minefields: An Approach to Studying Future-Choice Decisions", in Erwann Michel-Kerjan; Paul Slovic (orgs.), The Irrational Economist: Making Decisions in a Dangerous World. New York: Public Affairs, 21-29.

Hsee, Christopher; Hastie, Reid; Chen, Jingqiu (2008), "Hedonomics. Bridging Decision Research with Happiness Research", Perspectives on Psychological Science, 3(3), 224-243.

Huston, S. J. (2010), "Measuring Financial Literacy", Journal of Consumer Affairs, 44(2), 296-316.

Kahneman, Daniel (1973), Attention and Effort. Englewood Cliffs, New Jersey: Prentice-Hall, Inc.

Kahneman, Daniel (1999), “Objective Happiness”, in Daniel Kahneman; Ed Diener; Norbert Schwarz (orgs.), Well-being: The foundations of Hedonic Psychology. New York: Russel Sage Fundation, 3-25.

Kahneman, Daniel (2003), “A Perspective on Judgment and Choice: Mapping Bounded Rationality”, American Psychologist, 58, 697-720.

Kahneman, Daniel; Diener, Ed; Schwarz, Norbert (orgs.) (1999), Well-being: The Foundations of Hedonic psychology. New York: Russel Sage Fundation.

Kahneman, Daniel; Snell, Jackie (1990), "Predicting Utility”, in Robin Hogarth (org.), Insights in Decision Making: A Tribute to Hillel J. Einhorn. Chicago, IL: University of Chicago Press, 295-310.

Kahneman, Daniel; Snell, Jackie (1992), "Predicting a Changing Taste: Do People Know What they Will Like?”, Journal of Behavioral Decision Making, 5, 187-200.

Kahneman, Daniel; Tversky, Amos (1979), "Prospect Theory: An Analysis of Decision under Risk", Econometrica, 47(2), 263-291.

Kamleitner, Bernadette; Kirchler, Erich (2007), "Consumer Credit Use: A Process Model and Literature Review", Revue Européenne de Psychologie Appliquée, 57, 267-283.

Keren, Gideon; Roelofsma, Peter (1995), "Immediacy and Certainty in Intertemporal Choice", Organizational Behavior and Human Decision Processes, 63, 287-297.

Laibson, David (1997), "Golden Eggs and Hyperbolic Discounting”, The Quarterly Journal of Economics, 112(2), 443-478. 
Li, Shu; Su, Yin; Sun, Yan (2010), "The effect of pseudo-immediacy on intertemporal choices”, Journal of Risk Research, 13(6), 781-787.

Liberman, Nira; Trope, Yaacov; Wakslak, Cheryl (2007), "Construal Level Theory and Consumer Behavior", Journal of Consumer Psychology, 17(2), 113-117.

Lichtenstein, Sarah; Slovic, Paul (orgs.) (2006), The Construction of Preference. Cambridge, MA: Cambridge University Press.

Loewenstein, George (1996), "Out of Control: Visceral Influences on Behavior", Organizational Behavior and Human Decision Processes, 65, 272-92.

Loewenstein, George; Schkade, David (1999), “Wouldn't it Be Nice? Predicting Future Feelings”, in Daniel Kahneman; Ed Diener; Norbert Schwarz (orgs.), Well-being: The Foundations of Hedonic Psychology. New York: Russel Sage Fundation, 85-105.

Maule, John; Edland, Anne (1997), "The Effects of Time Pressure on Judgment and Decision Making”, in Rob Ranyard; W. Ray Crozier; Ola Svenson (orgs.), Decision Making. Cognitive Models and Explanations. London: Routledge, 189-204.

Maule, John; Hockey, Robert; Bdzola, Larissa (2000), "Effects of Time-pressure on Decision-making under Uncertainty: Changes in Affective State and Information Processing Strategy”, Acta Psychologica, 104, 283-301.

Oliveira, Armando Mónica (2007), “A 'heurística afectiva’ segundo P. Slovic: Entre manipulação e democracia”, in Carlos Henggeler Antunes; Luís Cândido Dias (orgs.), Decisão: perspectivas interdisciplinares. Coimbra: Imprensa da Universidade de Coimbra, 69-93.

Oliveira, José Miguel (2005), Racionalidade quebrada: A racionalidade ecológica das heurísticas rápidas e frugais. Dissertação de Doutoramento em Psicologia Cognitiva. Coimbra: FPCE-UC.

Payne, John; Bettman, James; Johnson, Eric (1993), The Adaptive Decision Maker. Cambridge, MA: Cambridge University Press.

Posner, Michael I. (org.) (1993), "Foundations of Cognitive Science”. Cambridge, MA: MIT Press.

Prelec, Drazen; Loewenstein, George (1998), "The Red and the Black: Mental Accounting of Savings and Debt", Marketing Science, 17(1), 4-28.

Ranyard, Rob; Craig, Gillian (1995), "Evaluating and Budgeting with Instalment Credit: An Interview Study”, Journal of Economic Psychology, 16, 449-467.

Rohrer, Doug; Pashler, Harold; Etchegaray, Jason (1998), “When Two Memories Can and Cannot Be Retrieved Concurrently", Memory e Cognition, 26(4), 731-739.

Samuelson, Paul (1937), "A Note on Measurement of Utility”, Review of Economic Studies, 4, 155-161.

Schwarz, Norbert (2004), "Meta-cognitive Experiences in Consumer Judgment and Decision Making”, Journal of Consumer Psychology, 14, 332-348.

Schwarz, Norbert (2007), "Attitude Construction: Evaluation in Context", Social Cognition, 25(3), 638-656. 
Schwarz, Norbert (2012), "Feelings-as-Information Theory", in Paul Van Lange, Arie Kruglanski; Tory Higgins (orgs.), Handbook of Theories of Social Psychology. Thousand Oaks, CA: Sage, 1, 289-308.

Schwarz, Norbert; Clore, Gerald (1983), "Mood, Misattribution, and Judgments of Well-being: Informative and Directive Functions of Affective States", Journal of Personality and Social Psychology, 45(3), 513-523.

Schwarz, Norbert; Sanna, Lawrence; Skurnik, Ian; Yoon, Carolyn (2007), "Metacognitive Experiences and the Intricacies of Setting People Straight: Implications for Debiasing and Public Information campaigns", Advances in Experimental Social Psychology, 39, 127-161. Schwarz, Norbert; Song, Hyunjin; Xu, Jing (2009), "When Thinking is Difficult: Metacognitive Experiences as Information”, in Michaela Wänke (org.), Social Psychology of Consumer Behavior. New York: Psychology Press, 201-223.

Shakespeare, William (2013), Henry IV, Part II, Act I, Scene II. Versão eletrónica, consultada a 29.03.13, em http://www.opensourceshakespeare.org/views/plays/ play_view.php? WorkID=henry4p2eAct=1eScene=2eScope=scene.

Simon, Herbert (1957), Models of Man: Social and Rational. Mathematical Essays of Rational Human Behavior in Society Detting. New York: Willey.

Simon, Herbert (1990), "Invariants of Human Behavior", Annual Review of Psychology, 41(1), 1-20.

Slovic, Paul; Finucane, Melissa; Peters, Ellen; McGregor, Donald (2007), "A heurística afectiva”, in Carlos Henggeler Antunes; Luís Cândido Dias (orgs.), Decisão: perspectivas interdisciplinares. Coimbra: Imprensa da Universidade de Coimbra, 25-68. Tradução de Miguel Oliveira.

Smith, Eliot; DeCoster, Jamie (2000), "Dual-process Models in Social and Cognitive Psychology: Conceptual Integration and Links to Underlying Memory Systems”, Personality and Social Psychology Review, 4(2), 108-131.

Soman, Dilip; Ainslie, George; Frederick, Shane; Li, Xiuping; Lynch, John; Moreau, Page; Mitchell, Andrew; Read, Daniel; Sawyer, Alan; Trope, Yaacov; Wertenbroch, Klaus; Zauberman, Gal (2005), “The Psychology of Intertemporal Discounting: Why Are Distant Events Valued Differently from Proximal Ones?”, Marketing Letters, 16, 347-360.

Song, Hyunjin; Schwarz, Norbert (2009), "If it's Difficult-to-pronounce, it Must Be Risky: Fluency, Familiarity, and Risk Perception”, Psychological Science, 20, 135-138.

St. Evans, Jonathan (2008), "Dual-Processing Accounts of Reasoning, Judgment, and Social Cognition", Annual Review of Psychology, 59, 255-78.

Stanovich, Keith; West, Richard (2000), "Individual Differences in Reasoning: Implications for the Rationality Debate”, Behavioral and Brain Sciences, 23, 645-665. Strack, Fritz; Deutsch, Roland (2004), "Reflective and Impulsive Determinants of Social Behavior", Personality and Social Psychology Review, 8(3), 220-247.

Strotz, Robert (1956), "Myopia and Inconsistency in Dynamic Utility Maximization”, Review of Economic Studies, 23(3), 165-180. 
Styles, Elizabeth (2005), Attention, Perception and Memory. An Integrated Introduction. Hove, UK: Psychology Press.

Svenson, Ola; Maule, John (orgs.) (1993), Time Pressure and Stress in Human Judgment and Decision Making. New York: Plenum Press

Sweller, John (1988), “Cognitive Load during Problem Solving: Effects on Learning”, Cognitive Science, 12, 257-285.

Szollos, Alex (2009), "Toward a Psychology of Chronic Time Pressure: Conceptual and Methodological Review", Time E Society, 18(2-3), 332-350.

Thaler, Richard (1980), "Toward a Positive Theory of Consumer Choice”, Journal of Economic Behavior and Organization, 1, 39-60.

Thaler, Richard (1985), "Mental Accounting and Consumer Choice", Marketing Science, 4(3), 199-214.

Thaler, Richard; Sunstein, Cass (2008), Nudge. Improving Decisions about Health, Wealth, and Happiness. New Haven: Yale University Press.

Trope, Yaacov; Liberman, Nira (2010), "Construal Level Theory of Psychological Distance”, Psychological Review, 117, 440-463.

Tversky, Amos; Kahneman, Daniel (1981), "The Framing of Decisions and the Psychology of Choice”, Science, 211, 453-458.

Van Boven, Leaf; Ashworth, Laurence (2007), "Looking Forward, Looking Back: Anticipation is More Evocative than Retrospection”, Journal of Experimental Psychology, 136(2), 289-300.

Vohs, Kathleen; Baumeister, Roy (orgs.) (2011), Handbook of Self-Regulation: Research, Theory, and Applications. New York: The Guilford Press [2.' ed.].

Von Neumann, John; Morgenstern, Oskar (1944), Theory of Games and Economic Behavior. Princeton, NJ: Princeton University Press [3. ${ }^{\text {a ed.]. }}$

Wang, Jing; Novemsky, Nathan; Dhar, Ravi; Baumeister, Roy (2010), “Trade-Offs and Depletion in Choice”, Journal of Marketing Research, 47(5), 910-919.

Weber, Bethany; Chapman, Gretchen (2005), "The Combined Effects of Risk and Time on Choice: Does Uncertainty Eliminate the Immediacy Effect? Does Delay Eliminate the Certainty Effect?", Organizational Behavior and Human Decision Processes, 96(2), 104-118.

Wegner, Daniel (1994), “Ironic Processes of Mental Control”, Psychological Review, 101(1), 34-52.

Wegner, Daniel (1997), "When the Antidote is the Poison: Ironic Mental Control Processes”, Psychological Sciences, 8, 148-50.

Willis, Lauren (2008), “Against Financial-Literacy Education”, Iowa Law Review, 94, 197-285.

Wilson, Timothy; Centerbar, David; Kermer, Deborah; Gilbert, Daniel (2005), “The Pleasures of Uncertainty: Prolonging Positive Moods in Ways People Do Not Anticipate", Journal of Personality and Social Psychology, 88, 5-21. 
Wilson, Timothy; Wheatley, Thalia; Meyers, Jonathan; Gilbert, Daniel; Axsom, Danny (2000), "Focalism: A Source of Durability Bias in Affective Forecasting", Journal of Personality and Social Psychology, 78, 821-836.

Wilson, Timothy; Gilbert, Daniel (2003), “Affective Forecasting”, in Mark Zanna (org.), Advances in Experimental Social Psychology. San Diego, CA: Academic Press, 35, 345-411.

Wilson, Timothy; Gilbert, Daniel (2005), "Affective Forecasting Knowing What to Want”, Current Directions in Psychological Science, 14(3), 131-134.

Wright, Peter (1974),"The Harassed Decision Maker: Time Pressure, Distractions and the Use Evidence", Journal of Applied Psychology, 59, 555-561. 
